\title{
Low-dose lipopolysaccharide inhibits neuronal apoptosis induced by cerebral ischemia/reperfusion injury via the PI3K/Akt/FoxO1 signaling pathway in rats
}

\author{
FAN HE ${ }^{1}$, NANNAN ZHANG ${ }^{1}$, YAN LV $^{1}$, WENHAO SUN ${ }^{2}$ and HUISHENG CHEN ${ }^{1}$ \\ ${ }^{1}$ Department of Neurology, General Hospital of Shenyang Military Area Command, Shenyang, Liaoning 110016; \\ ${ }^{2}$ Department of Neurology, The General Hospital of Tianjin Medical University, Tianjin 300020, P.R. China
}

Received September 7, 2017; Accepted September 3, 2018

DOI: $10.3892 / \mathrm{mmr} .2019 .9827$

\begin{abstract}
The present study aimed to investigate the effects of low-dose lipopolysaccharide (LPS) on ischemia/reperfusion (I/R)-induced brain injury, and to explore the mechanism of phosphoinositide 3-kinase (PI3K)/Akt/forkhead box protein (Fox)O1 signaling pathway. Male Sprague-Dawley rats were divided into control group (control), ischemia/reperfusion surgery group (I/R) and low-dose LPS treatment group (LPS). An I/R model was established and the hemodynamic parameters were recorded at the end of I/R injury. The brain tissues were observed by hematoxylin and eosin staining, immunohistochemistry and terminal deoxynucleotidyl-transferase-mediated dUTP nick end labeling staining. Microglia were treated with LPS following hypoxia/reoxygenation. The cellular viability was detected by 3-(4,5-dimethylthiazol-2-yl)-2,5-diphenyltetrazolium bromide assay. The apoptotic rate of microglia was detected using AnnexinV/propidium iodide staining. The expression of B-cell lymphoma (Bcl)-2, Bcl-2-associated X (Bax), and caspase-3 were detected by western blot analysis and reverse transcription-quantitative polymerase chain reaction. Akt, phosphorylated (p)-Akt, FoxO1 and p-FoxO1 expression were detected by western blotting. It was previously reported that, following I/R injury, neuronal cells were disorderly and brain injury markers (neuron-specific enolase and S100 $\beta$ ), inflammatory cytokines [interleukin (IL)-1 $\beta$, IL-6 and tumor necrosis factor- $\alpha$ ] levels were significantly upregulated. In the present study, the expression levels of Bax, caspase-3 Akt and p-Akt were significantly higher, while that of Bcl-2, FoxO1 and p-FoxO1 were significantly lower in the I/R group. LPS treatment significantly increased the viability of neuronal cells
\end{abstract}

Correspondence to: Professor Huisheng Chen, Department of Neurology, General Hospital of Shenyang Military Area Command, 83 Wenhua Road, Shenyang, Liaoning 110016, P.R. China E-mail: chszh@aliyun.com

Key words: lipopolysaccharide, ischemia/reperfusion, apoptosis, inflammatory phosphoinositide 3-kinase/protein kinase B/forkhead box protein $\mathrm{O} 1$ signaling pathway and decreased the rate of neuronal cell apoptosis. Following the addition of PI3K signaling pathway inhibitor LY294002 to microglia, LPS reduced the levels of activated Akt, increased the downstream regulatory gene phosphorylation of FoxO1 and reduced microglia apoptosis. It was concluded that LPS can alleviate I/R-induced brain injury, inhibit neuronal cells apoptosis and protect neuronal cells via the PI3K/Akt/FoxO1 signaling pathway.

\section{Introduction}

Cerebral ischemia reperfusion is one of the effective measures for the treatment of stroke; however, during cerebral ischemia/reperfusion (I/R), a large number of inflammatory factors are released from local ischemic tissue (1). These activated inflammatory cells can infiltrate local tissue, and activate the relevant inflammatory signaling pathway, such as the TLR4/NF- $\mathrm{B}$ signaling pathway, thereby resulting in severe inflammatory injury $(2,3)$. A recent study has demonstrated that I/R injury is accompanied with apoptosis (4), and apoptosis can increase with prolonged durations of ischemia (5). Therefore, the effects of apoptosis in I/R injury require further investigation.

Cerebral ischemia/hypoxia-induced neuronal apoptosis is regulated by the phosphoinositide 3 -kinase $(\mathrm{PI} 3 \mathrm{~K}) /$ serine/threonine protein kinase Akt pathway (6). Forkhead box O1 (FoxO1) is a downstream molecule of the P13K/Akt pathway (7). The PI3K/Akt/FoxO1 signaling pathway is a classical pathway that mediates cell survival, serves an important role in $\mathrm{I} / \mathrm{R}$ injury in the brain, intestine and liver, and is associated with inflammatory response and apoptosis (8-10).

Lipopolysaccharide (LPS) is a major component of the gram-negative bacteria cytoderm, which regulates inflammation and the immune system by activating the relevant receptor-mediated signaling pathway (11). Zhou et al (12) demonstrated that LPS induced PI3K/Akt phosphorylation as well as $\mathrm{NF}-\kappa \mathrm{B}$ activation and induced acute lung injury. High doses of LPS induce a strong inflammatory response, leading to fatal sepsis or septic shock; while low-dose LPS can induce a protective cross-tolerance state (13). One form of stimulus-induced tolerance leads to different types of 
injuries, including ischemia-reperfusion and trauma; however, autoimmune injury can exhibit protective effects $(14,15)$. Gong et al (16) demonstrated that in cerebral ischemic injury rats models, the neuroprotective effect of LPS pretreatment may occur via the regulation of inflammatory mediators, the induction of neuroprotective factors and the inhibition of proinflammatory cytokine release. The present study sought to investigate the effects of low-dose LPS on brain injury in cerebral I/R rats and on the protein expression of PI3K/AKT/FoxO1 pathway in rat models of cerebral I/R injury, and to investigate the protective effect of low-dose LPS against brain injury and its molecular mechanism, so as to provide evidence for treating cerebral I/R injury.

\section{Materials and methods}

Animals and ethics approval. A total of 30 male Sprague-Dawley rats weighing 260-280 g, aged 8 weeks, were obtained from Shenyang Military Region General Hospital Laboratory Animal Center [Shenyang, China; license No. SCXK (JUN) 2012--0002]. Animals were housed at a constant temperature $\left(22 \pm 1^{\circ} \mathrm{C}\right)$, with $50 \%$ relative humidity, and a $12-\mathrm{h}$ light/dark cycle. The rats had ad libitum access to food and autoclaved water. All animal procedures were approved by the Animal Experiments Ethics Committee of the General Hospital of Shenyang Military Region (Shenyang, China). Rats were randomly divided into three groups: The control group (control group; $\mathrm{n}=10$ ), intraperitoneal injection of saline for 3 days, $0.2 \mathrm{mg} / \mathrm{kg}, 1$ time/day; cerebral I/R group (I/R group; $\mathrm{n}=10$ ), established cerebral I/R injury model; LPS pretreatment $(\mathrm{n}=10)$, intraperitoneal injection of LPS (Escherichia coli 0128:B12, L2887, Sigma-Aldrich; Merck KGaA, Darmstadt, Germany) dissolved in saline, $0.2 \mathrm{mg} / \mathrm{kg} 3$ days before I/R (once a day).

Cells and groups. HAPI rat microglial cells were purchased from Bena Culture Collection (Jiangsu, China; cat. no. BNCC340723). Microglia were divided into the control group, I/R group, LPS+I/R group (LPS group) and PI3K inhibitor+LPS+I/R group (LY group). In the I/R group, I/R was simulated by hypoxia and reoxygenation; in the LPS group, LPS was administered prior to hypoxia and during reoxygenation; and, in the PI3K group, both LPS and PI3K inhibitor LY294002 $(2.5 \mu \mathrm{g} / \mathrm{kg}$; cat. no. B-0294; Beijing Solarbio Science \& Technology Co., Ltd., Beijing, China) were added prior to hypoxia and during reoxygenation.

Preparation of cerebral $I / R$ injury model in rats. Animal models were prepared in accordance with a previous method (17). Rats were intraperitoneally anesthetized with $40 \mathrm{mg} / \mathrm{kg}$ sodium pentobarbital. The right pterygopalatine artery and external carotid artery were ligated. A small cut was made on the external carotid artery by a syringe needle $\sim 0.5 \mathrm{~cm}$ from the ligation site. Then a nylon bolt was pushed into the internal carotid artery in the direction of the skull through the right external carotid artery incision. Common carotid artery bifurcation was taken as a marker and the nylon bolt pushed there. The middle cerebral artery was blocked when a slight resistance was felt in the depth of 18-20 mm. After blocking for $2 \mathrm{~h}$, the nylon thread was pulled out and the arterial stump was fastened securely; subcutaneous tissue and skin were sutured. In this way, cerebral I/R injury models were successfully established.

Establishment of hypoxia-reoxygenation model. A hypoxic solution was used to simulate the I/R injury process in microglia. The hypoxic solution consisted of $\mathrm{NaCl} 137 \mathrm{mM}$, $\mathrm{KCl} 12 \mathrm{mM}, \mathrm{MgCl}_{2} 0.49 \mathrm{mM}, \mathrm{CaCl}_{2}-2 \mathrm{H}_{2} \mathrm{O} 0.9 \mathrm{mM}$, HEPES $4 \mathrm{mM}$, deoxyglucose $10 \mathrm{mM}$, sodium sulfite $0.75 \mathrm{mM}$ and sodium lactate $20 \mathrm{mM}, \mathrm{pH}$ 6.5. During the hypoxia procedure, the cells were placed in $95 \% \mathrm{~N}_{2}$ and $5 \% \mathrm{CO}_{2}$ for $2 \mathrm{~h}$. During the reoxygenation process, cells were placed in normal culture medium (89\% DMEM+10\% $\mathrm{FBS}+1 \%$ resistance) and incubated for $4 \mathrm{~h}$ at $37^{\circ} \mathrm{C}$. The LPS group was administered $100 \mathrm{ng} / \mathrm{ml}$ LPS $(0.2 \mathrm{mg} / \mathrm{kg})$ for 3 days; LPS was administered again prior to hypoxia and during reoxygenation at $37^{\circ} \mathrm{C}$. In the PI3K group, both LPS and PI3K inhibitor LY294002 were added prior to hypoxia for $2 \mathrm{~h}$ and during reoxygenation for $4 \mathrm{~h}$ at $37^{\circ} \mathrm{C}$.

Neurological function score and changes in rat hemodynamics. Neurological function scores of rats in each group were assessed according to the Longa score scale (18). Longa scoring criteria were: 0 , no evident neurological deficit; 1 , left limb can flex but cannot extend when lifting the tails (mild); 2, unilateral circling, animals can maintain balance at resting state (moderate); 3, animals are unstable and incline to contralateral side at resting state (severe); and 4, no visible activity (extremely severe). The hemodynamics (rectal temperature, $\mathrm{pH}, \mathrm{PaCO}_{2}, \mathrm{PaO}_{2}$ and hemoglobin) were detected by the Datex-Ohmeda S/5 Entropy Module (Datex-Ohmeda Division, Instrumentarium Corp., Helsinki, Finland).

Specimen collection and processing. Arterial and venous blood samples were collected at $6 \mathrm{~h}$ after I/R; the rats were sacrificed by administration of an overdose of sodium pentobarbital. Then, the brain tissue was isolated from the rats of each group; 4 of the rats were used for triphenyl tetrazolium chloride (TTC) staining, 3 of them were fixed in $4 \%$ paraformaldehyde for $48 \mathrm{~h}$ at room temperature, and the other 3 were stored at $-80^{\circ} \mathrm{C}$ for western blotting and reverse transcription-quantitative polymerase chain reaction (RT-qPCR). The serum was obtained by centrifugation at $1,000 \mathrm{x}$ g for $10 \mathrm{~min}$ at $4^{\circ} \mathrm{C}$, and stored at $-80^{\circ} \mathrm{C}$.

TTC staining and infarct volume measurement. The brain tissues from 4 rats of each group were frozen at $-20^{\circ} \mathrm{C}$ for $20 \mathrm{~min}$. Each brain tissue was cut into $2 \mathrm{~mm}$ thick brain slices from the front to the rear with a rat brain slice mold, obtaining a total of 4 pieces. The brain tissues were transferred to a Petri dish containing $4 \%$ TTC solution, stained at $37{ }^{\circ} \mathrm{C}$ in the dark for $30 \mathrm{~min}$ and fixed with $4 \%$ paraformaldehyde solution at room temperature for $10 \mathrm{~min}$. In accordance with the method proposed by Swanson, the region of non-infarcted area on the infarct side and the area contralateral to the infarct of each brain slice were measured (19). Infarct volume was expressed as the percentage of the infarct volume to total contralateral volume: Contralateral infarct volume-the volume of non-infarcted area on the infarct side)/contralateral infarct volume $\mathrm{x} 100 \%$. 
Histopathological changes. Brain tissues were harvested and fixed in $4 \%$ paraformaldehyde for $48 \mathrm{~h}$ at room temperature. After $48 \mathrm{~h}$, specimens were dehydrated with $70,80,90,95$ and $100 \%$ ethanol, followed by clearing in xylene, embedding in paraffin and sectioning $(5-\mu \mathrm{m}$-thick sections). Sections were deparaffinized, transferred to xylene, rehydrated with a graded ethanol series, stained with hematoxylin for $3 \mathrm{~min}$, differentiated with $0.3 \%$ hydrochloric acid for $30 \mathrm{sec}$, diluted with aqueous ammonia for $1 \mathrm{~min}$ and counterstained with $0.5 \%$ eosin solution for $1 \mathrm{~min}$, all at room temperature. Sections were washed with distilled water after each step. Following staining, sections were dehydrated, rinsed, cleared in xylene, mounted, and the pathological changes of brain tissue were observed under a light microscope (5 fields were randomly selected; magnification, $\mathrm{x} 200)$.

Terminal deoxynucleotidyl-transferase-mediated dUTP nick end labeling (TUNEL) staining. The apoptosis of brain tissues was detected according to the protocols of the TUNEL kit (In situ cell death detection kit-POD; cat. no. 11684817910; Roche Diagnostics GmbH, Mannheim, Germany). Brain tissues were dehydrated, embedded, sectioned (5- $\mu$ m-thick sections) and incubated with $0.9 \% \mathrm{NaCl}$ for $5 \mathrm{~min}$ at room temperature, then rinsed twice with PBS, mixed with biotinylated nucleotides and terminal deoxynucleotidyl transferase, covered with plastic coverslips and incubated at $37^{\circ} \mathrm{C}$ for $60 \mathrm{~min}$. Following the addition of $50 \mu \mathrm{l}$ of TUNEL reaction mixture, samples were incubated for $60 \mathrm{~min}$ at $37^{\circ} \mathrm{C}$ in a humidified atmosphere in the dark. Following rinsing of the slides three times in PBS for 5 min each, samples were analyzed in a drop of PBS under a fluorescence microscope (5 fields were randomly selected; magnification, $\mathrm{x} 200)$.

Annexin V/propidium iodide (PI) staining. The Annexin V-PI (cat. no. BD 556547; BD Biosciences, Franklin Lakes, NJ, USA) method was used to detect the apoptosis rate (apoptosis rate=early apoptosis rate+late apoptosis rate) of microglia by flow cytometry. Cells were harvested with $0.05 \%$ trypsin (10 min at room temperature), washed three times with cold PBS $\left(4^{\circ} \mathrm{C}\right)$, and collected by centrifugation at $180 \mathrm{x}$ g for $5 \mathrm{~min}$ at $4^{\circ} \mathrm{C}$. Then, cells were resuspended in $200 \mu \mathrm{l}$ binding buffer and incubated with Annexin V $(10 \mu \mathrm{g} / \mathrm{ml})$ and PI $(10 \mu \mathrm{g} / \mathrm{ml})$ in the dark for $15 \mathrm{~min}$ at room temperature. The cells were detected using a flow cytometer (BD Biosciences). Data were analyzed using FlowJo version 10 software (FlowJo LLC, Ashland, OR, USA).

MTT assay. The cells in logarithmic growth phase were inoculated into 96-well culture plates at a density of 2,000-5,000 per well and $100 \mu \mathrm{l}$ cells per well. MTT solution ( $5 \mathrm{mg} / \mathrm{ml}$ ) $20 \mu \mathrm{l}$ was added to each well and the cells cultured for an additional $4 \mathrm{~h}$ at $37^{\circ} \mathrm{C}$. Then the supernatant was removed and $150 \mu \mathrm{l}$ DMSO was added per well. Cells were agitated for $10 \mathrm{~min}$ and the crystals fully dissolved. The optical density (OD) was measured at $570 \mathrm{~nm}$ with plate reader (Infinite 200 PRO, Tecan Group, Ltd., Mannedorf, Switzerland), and the cell survival rate was calculated and inhibition was investigated. Cell survival rate $=(O D$ value of the intervention group $/ O D$ value of the normal control group) $\mathrm{x} 100$.
Western blotting. The samples were ground and centrifuged in a pre-chilled RIPA Lysis Buffer (cat. no. P0013B; Beyotime Institute of Biotechnology) at 14,000 x $\mathrm{g}$ for $30 \mathrm{~min}$ at $4^{\circ} \mathrm{C}$. Protein concentration was determined using a BCA protein assay kit (23227; Thermo Fisher Scientific, Inc.). The supernatant of total protein $(40 \mu \mathrm{g})$ was extracted for $10 \%$ SDS-polyacrylamide gel electrophoresis and the protein was semi-dried for PVDF membrane transfer. The membranes were blocked with $5 \%$ skim milk powder for $2 \mathrm{~h}$ at room temperature and incubated overnight at $4^{\circ} \mathrm{C}$ with B-cell lymphoma 2 (Bcl-2; 1:1,000; cat. no. ab59348; Abcam, Cambridge, MA, USA), Bcl-2-associated X (Bax; 1:2,000; cat. no. ab32503; Abcam), caspase-3 (1:500 dilutions; cat. no. ab13847; Abcam), Akt (1:10,000; cat. no. ab179463; Abcam), FoxO1 (1:1,000 dilutions; cat. no. ab52857; Abcam), phosphorylated (p)-Akt (1:1,000 dilutions; cat. no. ab131443; Abcam) and p-FoxO1 (1:1,000; cat. no. ab131339; Abcam) antibodies. All the antibodies were diluted with antibody dilution buffer (cat. no. A1820; Beijing Solarbio Science \& Technology Co., Ltd.) followed by three washes and incubation with secondary antibody (goat anti-rabbit IgG/HRP antibody; 1:5,000; cat. no. ab6721; Abcam) for $1 \mathrm{~h}$ at room temperature. After four washes using TBST, cells were developed with ECL luminescence kit (32109; Thermo Fisher Scientific, Inc.) and gel imaging system, gray value was measured by using Quantity One software (version 4.6.8, Bio-Rad Laboratories, Inc., Hercules, CA, USA).

$R T$ - $q P C R$. Primers were designed according to the sequences of Bax, Bcl-2 and caspase 3 as reported in GenBank (https://www.ncbi.nlm.nih.gov/genbank/), and were synthesized by Sangon Biotech (Shanghai) Co.,Ltd.(Shanghai, China). Total RNA was isolated with TRIzol reagent (cat. no. 15596018; Invitrogen; Thermo Fisher Scientific, Inc., Waltham, MA, USA), RNA purity and quantification were tested by NanoDrop One Microvolume UV-Vis Spectrophotometer (cat. no. ND-ONE-W; Thermo Fisher Scientific, Inc.). Reversely transcribed into cDNA using 5X PrimeScript RT Master Mix $2 \mu \mathrm{l}$ (RR036A, Takara Biotechnology Co., Ltd., Dalian, China) and $8 \mu \mathrm{l}$ total RNA. The RT reaction was conducted at: $37^{\circ} \mathrm{C}$ for $15 \mathrm{~min}$ and then at $85^{\circ} \mathrm{C}$ for $5 \mathrm{sec}$. cDNA was then stored at $4^{\circ} \mathrm{C}$ until use. A SYBR Premix Ex Taq kit (cat. no. RR820A; Takara Biotechnology Co., Ltd., Dalian, China) was used for the detection according to the manufacturer's protocols. Initial denaturation at $95^{\circ} \mathrm{C}$ for $30 \mathrm{sec} ; 40$ cycles of $95^{\circ} \mathrm{C}$ for $5 \mathrm{sec}$ and $60^{\circ} \mathrm{C}$ for $30 \mathrm{sec}$. The relative gene expression data was analyzed with the $2^{-\Delta \Delta C q}$ method (20). The primers used for qPCR were: Bax forward, 5'-GTGGATACAGACTCCCCC-3', reverse, 5'-AGCGGCTGTTTGTCTGGA-3'; Bcl-2 forward, 5'-TGATAACCGGGAGATCGTGA-3', reverse, 5'-TCTCTG AAGACGCTGCTCAG3'; caspase-3 forward, 5'-TGAATG GAAACAACCAGT3', reverse 5'-TCAAGCACCTGACCC TTA3'; and GAPDH forward, 5'-AACTTTGGCATTGTG GAAGG3' and reverse 5'-CACATTGGGGGTAGGAACAC3'. The experiments were replicated three times.

Statistical analysis. Data were analyzed using SPSS version 19.0 statistical software (IBM Corp., Armonk, NY, USA) and measurement data were expressed as mean \pm standard deviation. Comparisons between groups were made using the 
A

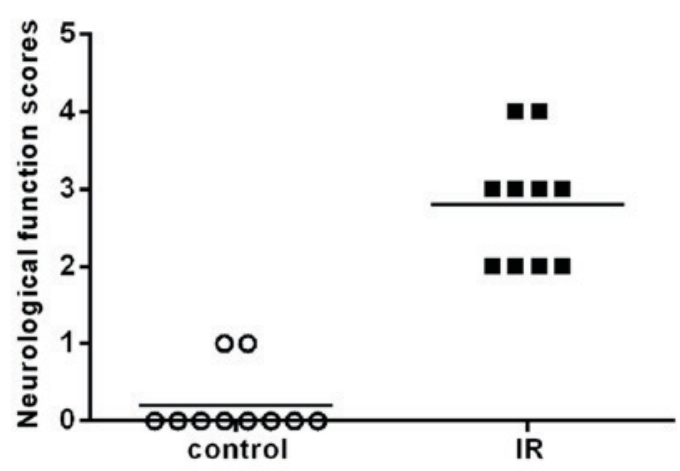

B
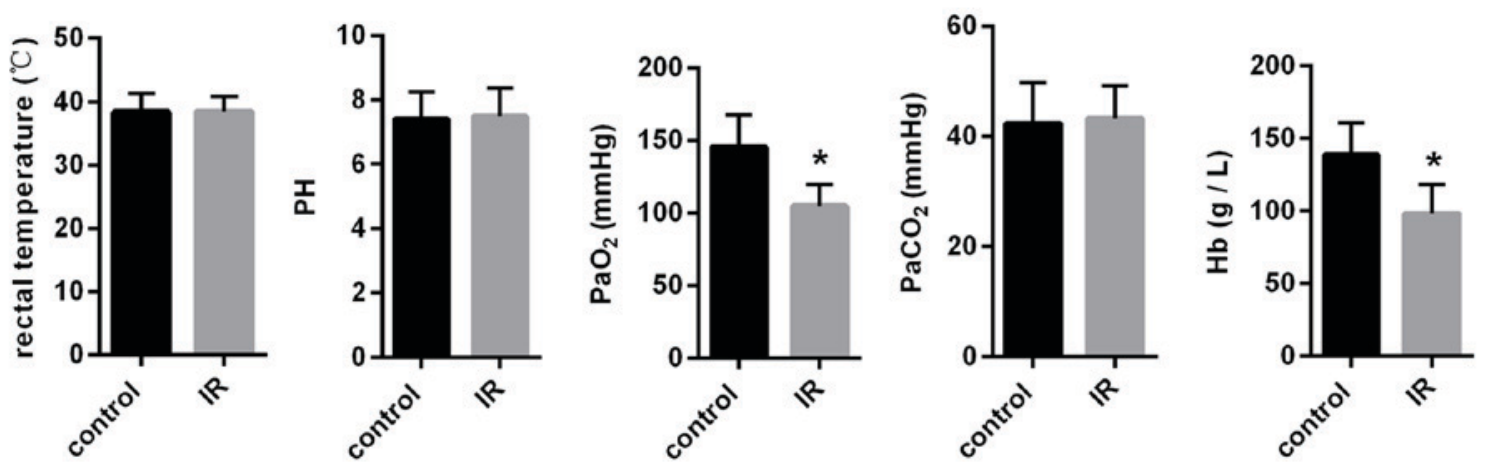

C
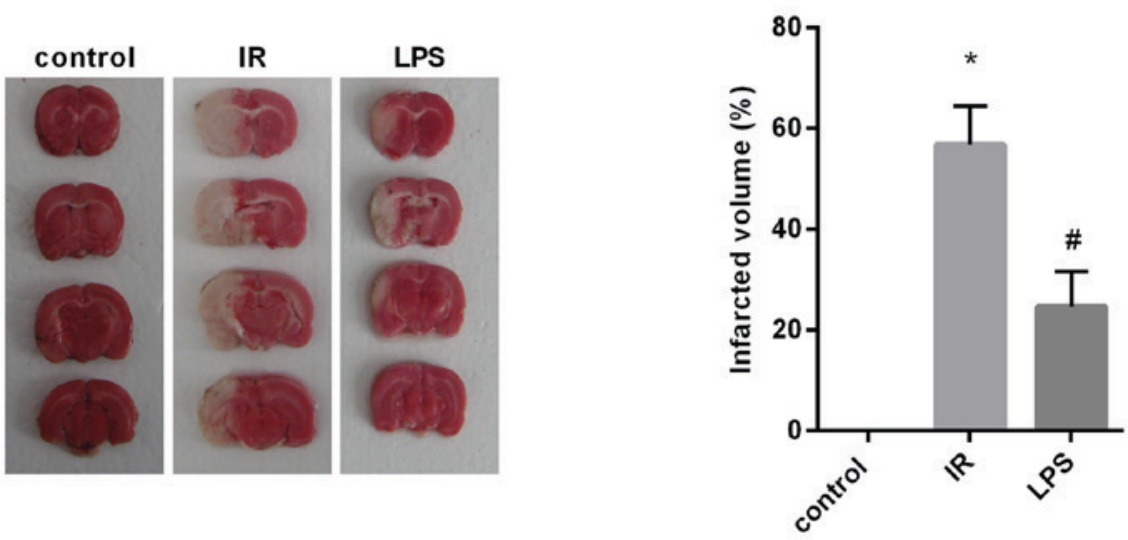

Figure 1. Establishment of I/R model in rats. (A) Neurological deficit scores and (B) hemodynamic changes in the control group and I/R groups. (C) TTC staining. ${ }^{\mathrm{P}}<0.05$ vs. the control group. ${ }^{*} \mathrm{P}<0.05$ vs. the $\mathrm{I} / \mathrm{R}$ group. Hb, hemoglobin; $\mathrm{I} / \mathrm{R}$, ischemia/reperfusion; $\mathrm{LPS}$, lipopolysaccharide; $\mathrm{PaCO}_{2}$, carbon dioxide partial pressure; $\mathrm{PaO}_{2}$, oxygen partial pressure; TTC, triphenyl tetrazolium chloride.

Student's t-test (two-tailed) or one-way analysis of variance with Tukey's post-test analysis. $\mathrm{P}<0.05$ was considered to indicate a statistically significant difference.

\section{Results}

Establishment of $I / R$ model in rats and identification of infarct area. All rats were conscious for 60-90 min following anesthesia. Rats in the control group did not show evident neurological deficits. Rats in the I/R group had different degrees of neurological abnormalities. Compared with the control group, neurological deficit scores significantly increased following $\mathrm{I} / \mathrm{R}(\mathrm{P}<0.05$; Fig. 1A). In the I/R group, rectal temperature, $\mathrm{pH}$ and arterial blood carbon dioxide partial pressure $\left(\mathrm{PaCO}_{2}\right)$ were stable; however, oxygen partial pressure $\left(\mathrm{PaO}_{2}\right)$ and hemoglobin was significantly decreased compared with the control (Fig. 1B). TTC staining demonstrated that the structure of the brain was normal in the control group; In the IR group, an evident infarct area was demonstrated, compared with the control group, the infarct volume was significant increased $(\mathrm{P}<0.05)$; the LPS group possessed an evident infarct area, compared with the IR group, the infarct volume was significant decreased. $(\mathrm{P}<0.05$; Fig. 1C). These results suggested that the $\mathrm{I} / \mathrm{R}$ model had been successfully established.

LPS protects I/R injury in rats. To determine the effect of LPS treatment following I/R injury, it was observed by hematoxylin and eosin staining that neuronal cells were arranged orderly in the control group, with clear boundaries and intact nuclei (Fig. 2A). Neuronal cells in the I/R group were disorderly, with unclear boundaries, myofiber rupture and fewer visible nuclei. The rats or the I/R group also found it difficult to maintain balance in a docile state (data not shown). In the LPS group, brain tissue injury was improved and the severity was alleviated compared with the I/R group; the neurological scores were reduced and every limb extended freely (Fig. 2B). These 
A

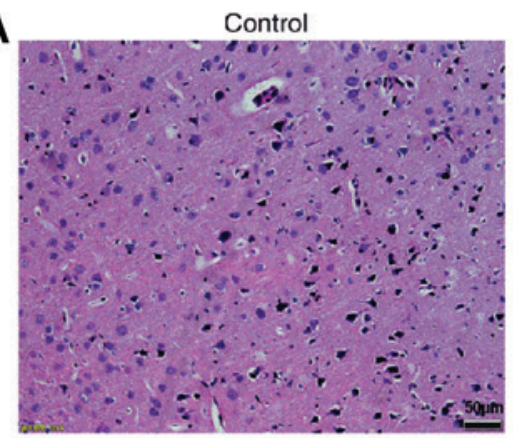

I/R

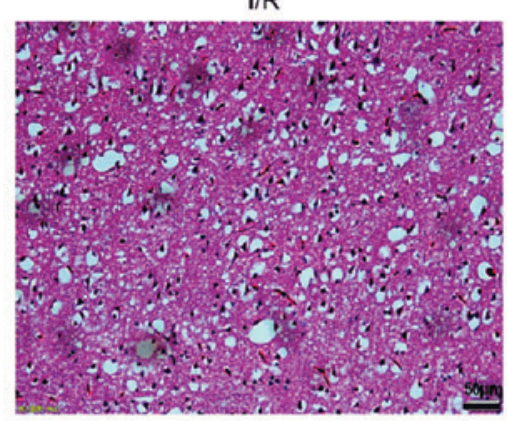

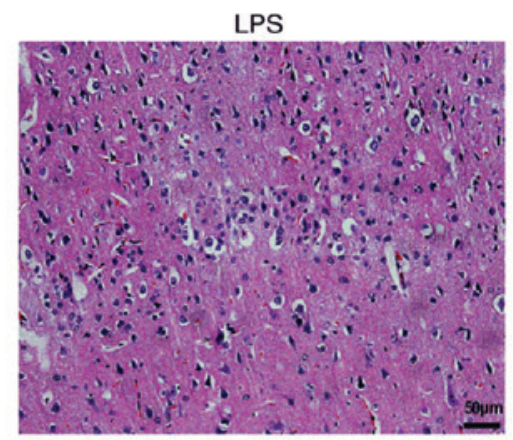

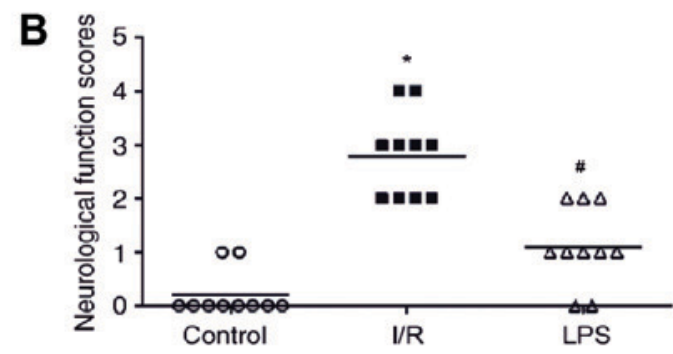

Figure 2. LPS protects against I/R injury in rats. (A) Hematoxylin and eosin staining. (B) Neurological deficit scores following LPS treatment. "P<0.05 vs. the control group. ${ }^{*} \mathrm{P}<0.05$ vs. the $\mathrm{I} / \mathrm{R}$ group. LPS, lipopolysaccharide; $\mathrm{I} / \mathrm{R}$, ischemia/reperfusion.

data indicated that LPS significantly attenuated brain tissue injury following I/R injury.

LPS protects brain tissue from I/R injury-induced apoptosis. To the effects of LPS on apoptosis following I/R injury were analyzed. It was observed that the number of TUNEL-positive cells had markedly increased in the I/R group compared with the control and that apoptotic cells had markedly decreased following LPS intervention (Fig. 3A). Next, the expression of apoptosis-related proteins and mRNA were examined. The expression of Bax and caspase-3 in brain tissue was significantly higher, while $\mathrm{Bcl}-2$ expression was significantly lower in the I/R group compared with in the control group $(\mathrm{P}<0.05)$. Following LPS intervention, Bax and caspase- 3 expression was significantly decreased and $\mathrm{Bcl}-2$ protein had increased compared with the I/R group (Fig. 3B and C). These results demonstrated that LPS treatment reduces neuronal cells apoptosis induced by cerebral I/R injury.

LPS inhibits neuronal apoptosis via the PI3K/Akt/FoxO1 signaling pathway following I/R injury. To ascertain whether the PI3K/Akt/FOXO1 signaling pathway mediated neuronal apoptosis, the expression levels of several key factors in the signaling pathway were detected in vitro and in vivo. In the I/R group, $\mathrm{PI} 3 \mathrm{~K}, \mathrm{Akt}$ and p-Akt expression increased, whereas FoxO1 and p-FoxO1 expression was significantly reduced compared with the control. LPS treatment significantly reversed the expression levels of these proteins toward normal levels in rats compared with the I/R group (Fig. 4A and B). These results suggested that protection of LPS against I/R injury induced cell apoptosis and may be mediated through the PI3K/Akt/FoxO1 signaling pathway. To further verify the mechanism, the cell viability in each group was detected with an MTT assay following reoxygenation. LPS treatment significantly enhanced the viability of microglia following hypoxia/reoxygenation compared with the I/R group ( $\mathrm{P}<0.05$; Fig. $4 \mathrm{C})$.
The apoptotic rate of the I/R group was $24.65 \%$ and that of the LPS group was $17.67 \%$. There was a significant difference between the I/R and LPS groups $(\mathrm{P}<0.05)$. In addition, LY294002, a PI3K pathway inhibitor, was used. The PI3K inhibitor was added to the LPS group, and the apoptotic rate was $23.55 \%(\mathrm{P}<0.05$; Fig. 4D and $\mathrm{E})$; a significant difference was observed compared with the LPS group. Akt was phosphorylated to p-Akt in the LPS group, the expression was increased, FoxO1 was inactivated and the expression of p-FoxO1 decreased, which was statistically different compared with the $\mathrm{I} / \mathrm{R}$ group $(\mathrm{P}<0.05)$. Following the addition of PI3K inhibitor, the role of LPS was inhibited, as the levels of p-Akt and p-FoxO1 were significantly decreased and increased, respectively (Fig. 4F and G). The data indicated that low-dose LPS can reduce I/R-induced brain injury and prevent brain injury through the PI3K/Akt/FOXO1 signaling pathway.

\section{Discussion}

Ischemic stroke is the second most common type critical disease worldwide and leads to mortality and permanent dysfunction, including cricopharyngeal dysfunction $(21,22)$. Brain injury induced by cerebral ischemia is the cause of exacerbations and damage mechanisms, including inflammatory response, oxidative stress, apoptosis and mitochondrial dysfunction (23-26). With immediate ischemia and reperfusion, a large number of inflammatory factors and oxygen free radicals are released, caused by the infiltration of inflammatory cells and neutrophil degranulation, while the formation of apoptotic bodies is stimulated $(27,28)$. The present study observed the effects of low-dose LPS on I/R-induced brain injury, and the results demonstrated that low-dose LPS suppressed I/R-induced morphological changes, decreased inflammatory factor expression and diminished apoptosis. The results also demonstrated that LPS decreased I/R-induced protein expression of PI3K/Akt/FoxO1 pathway and suggested 
A
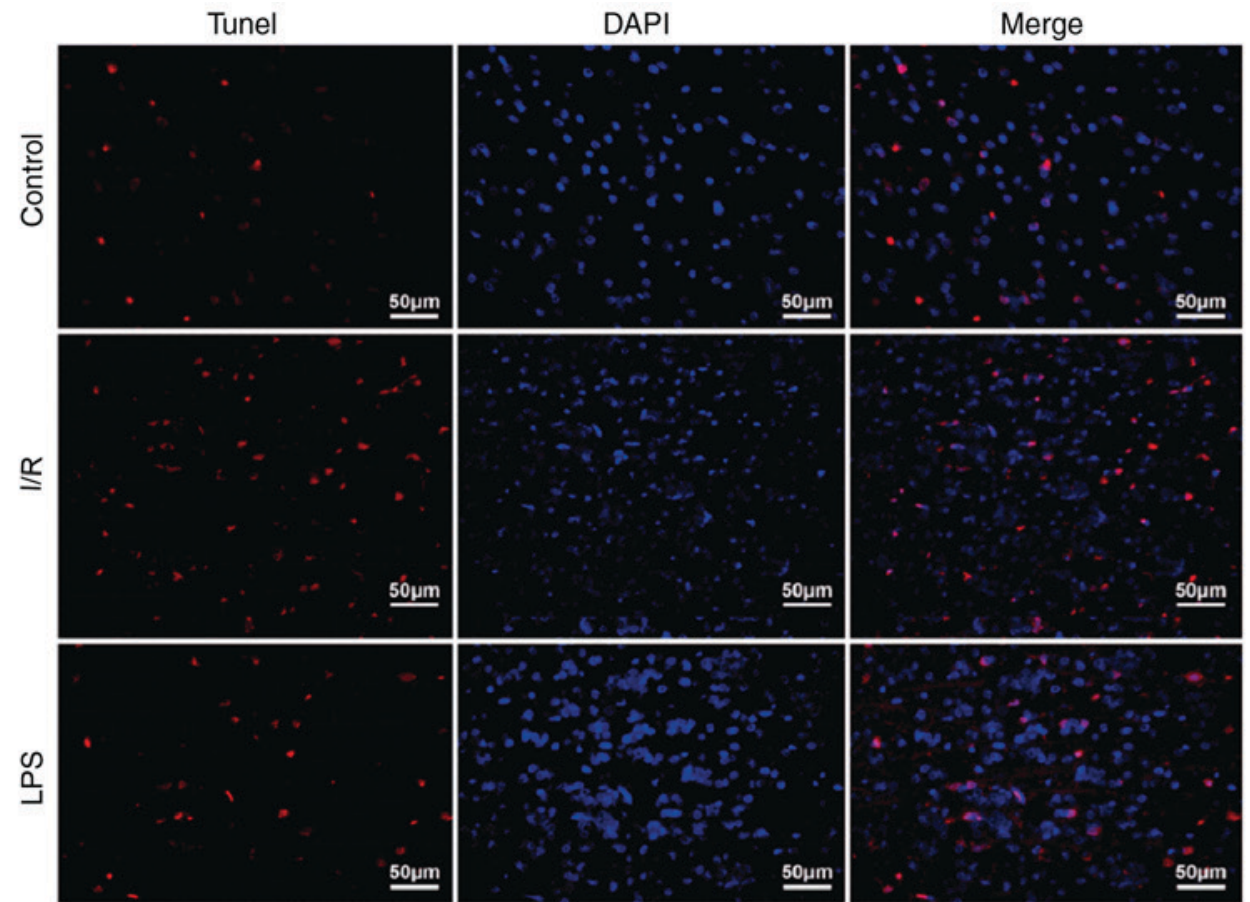

$\mathrm{B}$
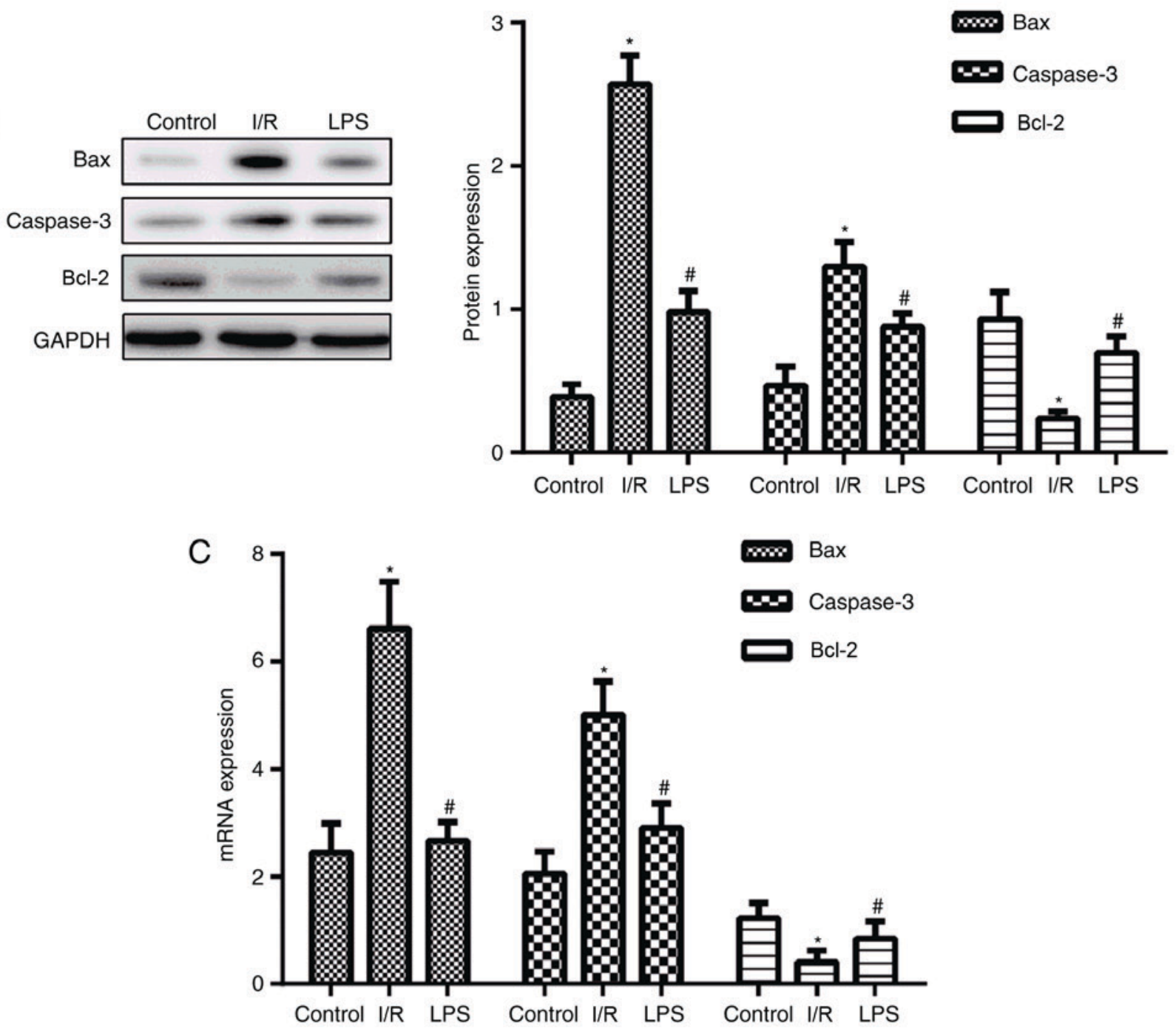

Figure 3. LPS protected brain tissue from I/R injury-produced apoptosis. (A) The apoptosis of brain tissues as detected by TUNEL. Scale bar=50 $\mu \mathrm{m}$. (B) The expression of Bcl-2, Bax and caspase-3 as detected by western blotting. (C) The expression of Bcl-2, Bax and caspase- 3 as detected by reverse transcription-quantitative polymerase chain reaction. " $\mathrm{P}<0.05$ vs. the control group; ${ }^{\sharp} \mathrm{P}<0.05 \mathrm{vs}$. I/R group. LPS, lipopolysaccharide; I/R, ischemia/reperfusion; TUNEL, terminal deoxynucleotidyl-transferase-mediated dUTP nick end labeling; Bcl-2, B-cell lymphoma 2; Bax, Bcl-2-associated X. 

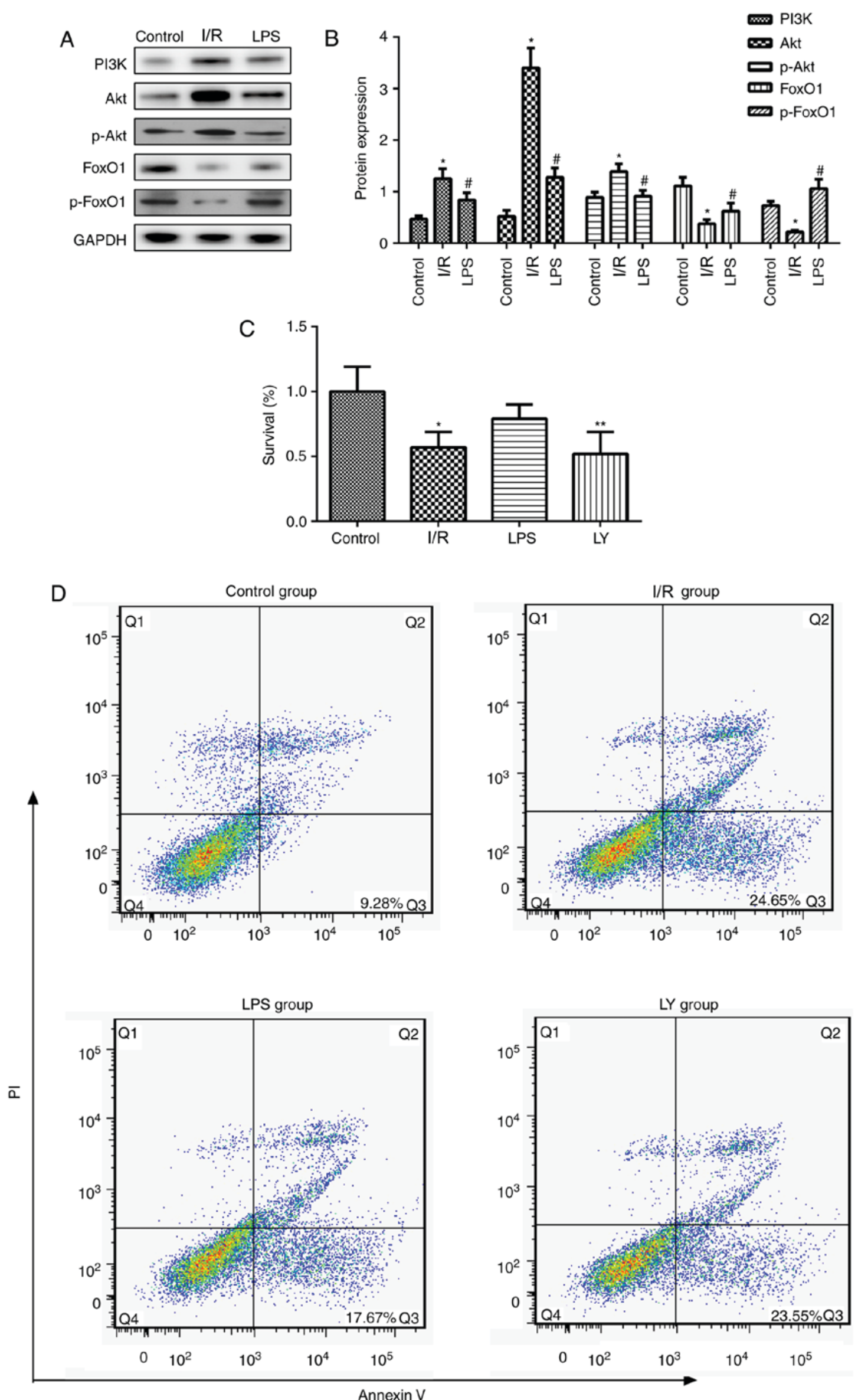

Figure 4. LPS inhibits neuronal apoptosis via the PI3K/Akt/FoxO1 signaling pathway following I/R injury. (A and B) Expression of PI3K, Akt, P-Akt, FoxO1 and p-FoxO1 as detected by western blotting. (C) Cell viability as detected by an MTT assay. (D) Apoptotic rate of microglia as detected by Annexin V and PI staining. 


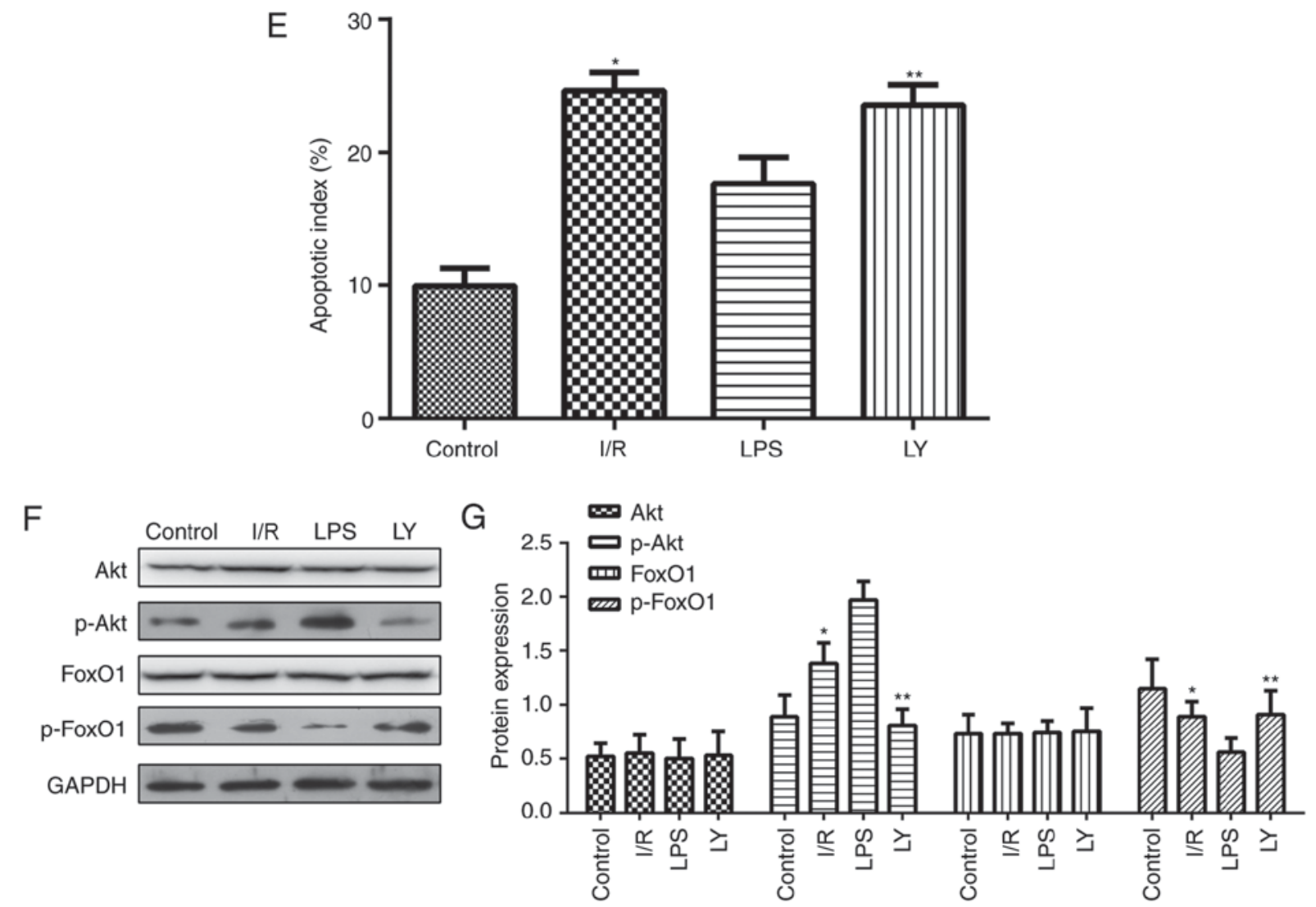

Figure 4. Continued. (E) Apoptotic rate of microglia as detected by Annexin V and PI staining. (F and G) Akt, p-Akt, FoxO1 and p-FoxO1 as detected by western blotting. " $\mathrm{P}<0.05$ vs. the control; ${ }^{\mathrm{P}} \mathrm{P}<0.05$ vs. I/R group; ${ }^{* *} \mathrm{P}<0.05$ vs. LPS group. FoxO1, forkhead box protein O1; I/R, ischemia/reperfusion; LPS, lipopolysaccharide; LY, LY294002 (2.5 $\mu \mathrm{g} / \mathrm{kg})$; PI3K, phosphoinositide 3-kinase; p-, phospho-; PI, propidium iodide.

that low-dose LPS relieved brain injury following I/R injury, possibly through the PI3K/Akt/FoxO1 pathway.

LPS as a small molecule material, can pass through the blood-brain barrier and gathers in the central nervous system (29). Low-dose LPS can induce the body to exhibit cross-immune tolerance, serving a protective effect on brain injury (30). The present study reported that LPS can significantly enhance the tolerance against cerebral I/R injury and protect against subarachnoid hemorrhage and may improve the prognosis of neurological function. Rosenzweig et al (31) demonstrated that pretreatment with $0.2 \mathrm{mg} / \mathrm{kg}$ LPS significantly reduced the cerebral ischemic inflammatory response. Ding and Li (32) identified that low-dose LPS pretreatment can reduce the production of pro-inflammatory cytokines, including tumor necrosis factor $\alpha$, and reduce glial cell inflammatory injury. In the present study, a rat model of cerebral I/R was established. Following low-dose LPS pretreatment, injury to the brain tissue was reduced, suggesting that low-dose LPS can inhibit the inflammatory response in cerebral $\mathrm{I} / \mathrm{R}$.

In the process of cerebral I/R, an abnormal interaction occurs among inflammatory, vascular endothelial and nerve cells, and a large number of soluble inflammatory factors are released, which. mediate apoptosis-induced intracellular calcium overload (33). In the early stage of reperfusion, neutrophils and vascular endothelial cells release chemokines, leading to vascular dysfunction, tissue injury and finally apoptosis is induced. To further observe the effect of low-dose LPS on the apoptosis of I/R injury, TUNEL was used to detect the apoptosis of neurons in the cortical ischemic area. The results of the present study revealed that the apoptosis of microglia of the I/R group was significantly higher compared with the control and LPS groups, while the LPS group exhibited significantly fewer apoptotic cells compared with the I/R group. Furthermore, low-dose LPS could significantly reduce Bax and caspase-3 expression levels following I/R injury, indicating that low-dose LPS exerted protective effects against cerebral I/R injury in rats via an anti-apoptotic mechanism.

Previous studies have demonstrated that the PI3K/AKT signaling pathways are involved in neuronal apoptosis in cerebral ischemia (34-36). The activation of the intrinsic pathway and external inflammatory can drive the AKT-mediated cell survival pathway, activate PI3K, connect the intracellular C-terminal of the $\alpha$-amino-3-hydroxy-5-methyl-4-isoxazole propionic acid receptor, activate its downstream kinase Akt (Ser473) residue phosphorylation and induce the downstream protein FoxO1 to the cell membrane translocation $(37,38)$. Phosphorylation of Ser9 causes FoxO1 inactivation and phosphorylated $\beta$-catenin promotes cell survival $(39,40)$. The results of the present study demonstrated that low-dose LPS treatment significantly reduced the apoptotic rate in rats following I/R or in cells following hypoxia/reoxygenation. The PI3K/Akt/FoxO1 signaling pathway serves an important role. Low-dose LPS in the I/R model can be used to activate PI3K and AKT phosphorylation, increase p-Akt expression and decrease p-FoxO1 expression. To further verify the mechanism, LY294002 was used in vitro. The data demonstrated that 
following the addition of the inhibitor, low-dose LPS could not activate AKT phosphorylation and its inhibitory effect on apoptosis was also limited. The data indicated that low-dose LPS can reduce I/R-induced brain injury and prevent brain injury through the PI3K/Akt/FOXO1 signaling pathway.

In summary, LPS ameliorated morphological changes in the hippocampus, reduced inflammatory factor expression and diminished apoptosis following I/R injury. Its mechanism may be exerted through the PI3K/Akt/FoxO1 pathway, which provide a new theoretical basis for clinical treatment of I/R injury.

\section{Acknowledgements}

Not applicable.

\section{Funding}

The present study was supported by the China Postdoctoral Science Foundation (grant no. 2016M593025).

\section{Availability of data and materials}

All data generated or analyzed during the present study are included in this published article.

\section{Authors' contributions}

FH, NZ, YL and WS performed the qRT-PCR and collected data. NZ and YL participated in samples collection. WS guided the collection of samples. HC contributed to the major part of funding support. FH and $\mathrm{HC}$ designed the study. FH wrote the manuscript. All authors read and approved the final manuscript.

\section{Ethics approval and consent to participate}

The present study was approved by the Animal Experiments Ethics Committee of the General Hospital of Shenyang Military Region (Shenyang, China).

\section{Patient consent for publication}

Not applicable.

\section{Competing interests}

The authors declare that they have no competing interests.

\section{References}

1. Bhaskar S, Stanwell P, Cordato D, Attia J and Levi C: Reperfusion therapy in acute ischemic stroke: Dawn of a new era? BMC Neurol 18: 8, 2018

2. Wang Q, Dai P, Bao H, Liang P, Wang W, Xing A and Sun J: Anti-inflammatory and neuroprotective effects of sanguinarine following cerebral ischemia in rats. Exp Ther Med 13: 263-268, 2017.

3. Wang SL, Duan L, Xia B, Liu Z, Wang Y and Wang GM: Dexmedetomidine preconditioning plays a neuroprotective role and suppresses TLR4/NF- $\mathrm{BB}$ pathways model of cerebral ischemia reperfusion. Biomed Pharmacother 93: 1337-1342, 2017.

4. Bai S, Sun Y, Wu L, Wu Z and Fang M: Tripotolide ameliorates inflammation and apoptosis induced by focal cerebral ischemia/reperfusion in rats. Zhejiang Da Xue Xue Bao Yi Xue Ban 45: 493-500, 2016 (In Chinese).
5. Sasaki H, Galang N and Maulik N: Redox regulation of NF-kappaB and AP-1 in ischemic reperfused heart. Antioxid Redox Signal 1: 317-324, 1999.

6. Zhang L, Qu Y, Tang J, Chen D, Fu X, Mao M and Mu D $\mathrm{PI} 3 \mathrm{~K} / \mathrm{Akt}$ signaling pathway is required for neuroprotection of thalidomide on hypoxic-ischemic cortical neurons in vitro. Brain Res 1357: 157-165, 2010.

7. Cui XB, Wang C, Li L, Fan D, Zhou Y, Wu D, Cui QH, Fu FY and $\mathrm{Wu}$ LL: Insulin decreases myocardial adiponectin receptor 1 expression via PI3K/Akt and FoxO1 pathway. Cardiovasc Res 93: 69-78, 2012.

8. Cao YT, Zhou L, Wu HT, Li XM, Huang WT, Chen XL, Li C and Tao L: The clinical characteristics and treatment outcomes of 386 patients with hypopharyngeal cancer. Zhonghua Er Bi Yan Hou Tou Jing Wai Ke Za Zhi 51: 433-439, 2016 (In Chinese).

9. Huang CY, Hsiao JK, Lu YZ, Lee TC and Yu LC: Anti-apoptotic PI3K/Akt signaling by sodium/glucose transporter 1 reduces epithelial barrier damage and bacterial translocation in intestinal ischemia. Lab Invest 91: 294-309, 2011.

10. Chu SF, Zhang Z, Zhang W, Zhang MJ, Gao Y, Han N, Zuo W, Huang HY and Chen NH: Upregulating the Expression of Survivin-HBXIP Complex Contributes to the Protective Role of IMM-H004 in transient global cerebral ischemia/reperfusion. Mol Neurobiol 54: 524-540, 2017.

11. Wyns H, Plessers E, De Backer P, Meyer E and Croubels S: In vivo porcine lipopolysaccharide inflammation models to study immunomodulation of drugs. Vet Immunol Immunopathol 166: 58-69, 2015.

12. Zhou B, Weng G, Huang Z, Liu T and Dai F: Arctiin prevents LPS-induced acute lung injury via inhibition of PI3K/AKT signaling pathway in mice. Inflammation, 2018 (Epub ahead of print).

13. Delayre-Orthez C, de Blay F, Frossard N and Pons F: Dose-dependent effects of endotoxins on allergen sensitization and challenge in the mouse. Clin Exp Allergy 34: 1789-1795, 2004.

14. Ronco C: Lipopolysaccharide (LPS) from the cellular wall of Gram-negative bacteria, also known as endotoxin, is a key molecule in the pathogenesis of sepsis and septic shock. Preface. Blood Purif 37 (Suppl 1): S1, 2014.

15. Dai Y, Jia P, Fang Y, Liu H, Jiao X, He JC and Ding X: miR-146a is essential for lipopolysaccharide (LPS)-induced cross-tolerance against kidney ischemia/reperfusion injury in mice. Sci Rep 6: 27091, 2016.

16. Gong G, Huang Y, Yuan LB, Hu L and Cai L: Requirement of LRG in endotoxin-mediated brain protection. Xi Bao Yu Fen $\mathrm{Zi}$ Mian Yi Xue Za Zhi 27: 865-867, 2011 (In Chinese).

17. Augestad IL, Nyman AKG, Costa AI, Barnett SC, Sandvig A, Håberg AK and Sandvig I: Effects of neural stem cell and olfactory ensheathing cell co-transplants on tissue remodelling after transient focal cerebral ischemia in the adult rat. Neurochem Res 42: 1599-1609, 2017.

18. Liu YZ, Wang C, Wang Q, Lin YZ, Ge YS, Li DM and Mao GS: Role of fractalkine/CX3CR1 signaling pathway in the recovery of neurological function after early ischemic stroke in a rat model. Life Sci 184: 87-94, 2017.

19. Swanson RA, Morton MT, Tsao-Wu G, Savalos RA, Davidson C and Sharp FR: A semiautomated method for measuring brain infarct volume. J Cereb Blood Flow Metab 10: 290-293, 1990.

20. Livak KJ and Schmittgen TD: Analysis of relative gene expression data using real-time quantitative PCR and the 2(-Delta Delta C(T)) method. Methods 25: 402-408, 2001.

21. Chancellor BK and Ishida K: New standards of care in ischemic stroke. J Neuroophthalmol 37: 320-331, 2017.

22. Yang H, Yi Y, Han Y and Kim HJ: Characteristics of cricopharyngeal dysphagia after ischemic stroke. Ann Rehabil Med 42: 204-212, 2018.

23. Kim E, Tolhurst AT and Cho S: Deregulation of inflammatory response in the diabetic condition is associated with increased ischemic brain injury. J Neuroinflammation 11: 83, 2014.

24. Yuksel S, Tosun YB, Cahill J and Solaroglu I: Early brain injury following aneurysmal subarachnoid hemorrhage: Emphasis on cellular apoptosis. Turk Neurosurg 22: 529-533, 2012.

25. Huang L, Wan J, Chen Y, Wang Z, Hui L, Li Y, Xu D and Zhou W: Inhibitory effects of p38 inhibitor against mitochondrial dysfunction in the early brain injury after subarachnoid hemorrhage in mice. Brain Res 1517: 133-140, 2013.

26. Li T, Luo N, Du L, Zhou J, Zhang J, Gong L and Jiang N: Tumor necrosis factor-alpha plays an initiating role in extracorporeal circulation-induced acute lung injury. Lung 191: 207-214, 2013.

27. Zhang C, Wang JG, Zou WX and Tian WJ: Clinical study on naomaltal capsule in treating lschemic cerebral infarction. Guide Chinese Medicine 6: 74, 2008 
28. Halladin NL: Oxidative and inflammatory biomarkers of ischemia and reperfusion injuries. Dan Med J 62: B5054, 2015.

29. Sperandeo P, Martorana AM and Polissi A: Lipopolysaccharide biogenesis and transport at the outer membrane of Gram-negative bacteria. Biochim Biophys Acta Mol Cell Biol Lipids 1862: 1451-1460, 2017.

30. Li ZX, Li QY, Qiao J, Lu CZ and Xiao BG: Granulocyte-colony stimulating factor is involved in low-dose LPS-induced neuroprotection. Neurosci Lett 465: 128-132, 2009.

31. Rosenzweig HL, Minami M, Lessov NS, Coste SC, Stevens SL, Henshall DC, Meller R, Simon RP and Stenzel-Poore MP: Endotoxin preconditioning protects against the cytotoxic effects of TNFalpha after stroke: A novel role for TNFalpha in LPS-ischemic tolerance. J Cereb Blood Flow Metab 27: 1663-1674, 2007.

32. Ding Y and Li L: Lipopolysaccharide preconditioning induces protection against lipopolysaccharide-induced neurotoxicity in organotypic midbrain slice culture. Neurosci Bull 24: 209-218, 2008.

33. Qin T, Li N, Tan XF, Zheng JH, Tao R and Chen MH: Works on heart, how about brain? Effect of hyperkalemia on focal cerebral ischemia/reperfusion injury in rats. Eur Rev Med Pharmacol Sci 22: 2839-2846, 2018.

34. Jiang EP, Wang SQ, Wang Z, Yu CR, Chen JG and Yu CY: Effect of schisandra chinensis lignans on neuronal apoptosis and p-AKT expression of rats in cerebral ischemia injury model. Zhongguo Zhong Yao Za Zhi 39: 1680-1684, 2014 (In Chinese).

35. Zhong JB, Li X, Zhong SM, Liu JD, Chen CB and Wu XY: Knockdown of long noncoding antisense RNA brain-derived neurotrophic factor attenuates hypoxia/reoxygenation-induced nerve cell apoptosis through the BDNF-TrkB-PI3K/Akt signaling pathway. Neuroreport 28: 910-916, 2017.
36. Li W, Yang Y, Hu Z, Ling S and Fang M: Neuroprotective effects of DAHP and Triptolide in focal cerebral ischemia via apoptosis inhibition and PI3K/Akt/mTOR pathway activation. Front Neuroanat 9: 48, 2015

37. Theofilatos D, Fotakis P, Valanti E, Sanoudou D, Zannis V and Kardassis D: HDL-apoA-I induces the expression of angiopoietin like 4 (ANGPTL4) in endothelial cells via a PI3K/AKT/FOXO1 signaling pathway. Metabolism 87: 36-47, 2018.

38. Mattoon DR, Lamothe B, Lax I and Schlessinger J: The docking protein Gab1 is the primary mediator of EGF-stimulated activation of the PI-3K/Akt cell survival pathway. BMC Biol 2: 24, 2004.

39. Huang J, Kodithuwakku ND, He W, Zhou Y, Fan W, Fang W, $\mathrm{He} \mathrm{G}, \mathrm{Wu} \mathrm{Q}, \mathrm{Chu} \mathrm{S}$ and $\mathrm{Li} \mathrm{Y}$ : The neuroprotective effect of a novel agent $\mathrm{N} 2$ on rat cerebral ischemia associated with the activation of PI3K/Akt signaling pathway. Neuropharmacology 95: 12-21, 2015

40. Perkinton MS, Sihra TS and Williams RJ: Ca(2+)-permeable AMPA receptors induce phosphorylation of cAMP response element-binding protein through a phosphatidylinositol 3-kinase-dependent stimulation of the mitogen-activated protein kinase signaling cascade in neurons. J Neurosci 19: 5861-5874, 1999.

This work is licensed under a Creative Commons Attribution-NonCommercial-NoDerivatives 4.0 International (CC BY-NC-ND 4.0) License. 